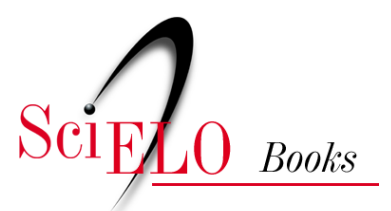

\title{
11. A complexidade da assistência às urgências e emergências e o caso do estado do Rio de Janeiro
}

\author{
Gisele O’Dwyer
}

\section{SciELO Books / SciELO Livros / SciELO Libros}

O'DWYER, G. A complexidade da assistência às urgências e emergências e o caso do estado do Rio de Janeiro. In: UGÁ, M.A.D., et al., (orgs.). A gestão do SUS no âmbito estadual: o caso do Rio de Janeiro [online]. Rio de Janeiro: Editora FIOCRUZ, 2010, pp. 279-298. ISBN: 978-85-7541-592-4. Available from: doi: 10.7476/9788575415924.0013. Also available in ePUB from: http://books.scielo.org/id/c2hxb/epub/uga-9788575415924.epub.

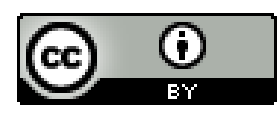

All the contents of this work, except where otherwise noted, is licensed under a Creative Commons Attribution $\underline{4.0 \text { International license. }}$

Todo o conteúdo deste trabalho, exceto quando houver ressalva, é publicado sob a licença Creative Commons Atribição 4.0. 


\title{
A COMplexidade da AssistêncIA Às URGÊNCIAS E EMERGÊNCIAS E O Caso do Estado do Rio de Janeiro
}

\author{
Gisele O'Dwyer
}

$\mathrm{E}_{\text {ste capítulo pretende apresentar algumas considerações sobre o atual caos dos }}$ serviços de assistência às urgências e emergências no estado do Rio de Janeiro. Começo relacionando a estrutura e organização do Sistema Único de Saúde (SUS) com o impacto produzido nesse nível de assistência. O SUS, como política pública, constitui uma arena de conflitos em que atuam diversos grupos de interesse, que ora contribuem para dar sustentação e legitimidade à política, ora estabelecem tensões para sua implementação (Viana, Faria \& Pacífico, 2006). Esse é um dos aspectos de impacto, que trata de questões relacionais e organizacionais. Vou enfatizar o aspecto mais estrutural do SUS por acreditar que as 'insuficiências' vividas pelos profissionais vêm influenciando uma prática que tensiona a organização dos serviços e a relação profissional/usuário.

Pode-se dizer que a insuficiência de recursos chega a inviabilizar o funcionamento de serviços locais, sobrecarregando de maneira sobre-humana os profissionais de saúde e responsabilizando-os, às vezes de maneira espalhafatosa, pelas falhas resultantes do estrangulamento do sistema de atenção à saúde. Nos anos 90, o Brasil tratou sua força de trabalho na área da saúde de forma predatória, pela defasagem crescente de seus salários, pela quase inexistência de recursos para atender ao crescimento da demanda por atenção médica, ou ao menos para repor o contingente de profissionais em função de mortes ou aposentadorias, e pela exigência de uma crescente produtividade desacompanhada de condições mínimas de trabalho adequadas para o desempenho de suas funções. O reflexo dessa situação tem sido dramático, sobretudo entre os médicos (Luz, 2007).

A seguir apresento as ações de regularização desse setor a partir das políticas propostas nos últimos anos. Faço não só uma descrição, mas uma discreta análise do potencial regulatório e de seu conteúdo. Finalmente particularizo o caso do estado do Rio de Janeiro usando material empírico de uma pesquisa realizada em 2005/2006.

Espero com este breve texto apontar os principais nós gerenciais e, mais que isso, sensibilizar os leitores para a importância de entender os serviços como espaços construídos 
socialmente, sendo ao mesmo tempo meio e resultado de uma dualidade (caráter duplo) e não de um dualismo (caráter oposto) entre estrutura e agente humano. Aceitar a dualidade consiste em negar uma versão em que a estrutura seria uma força geradora de práticas que engessam a capacidade de mobilização do agente humano e outra versão em que haveria um privilégio da intencionalidade e subjetividade nas práticas sociais. Dito de outra forma, aceitar a prática dos serviços como um processo social sem deixar de atribuir ao profissional capacidade de reflexão, inovação e superação, já que no SUS também existem experiências bem-sucedidas de encontros solidários entre profissionais e usuários.

\section{O QUANTO AS INSUFICIÊNCIAS DO SUS INTERFEREM NOS Problemas Vividos nas Emergências}

Ao analisar o SUS a partir de seus princípios de integralidade e equidade e de sua diretriz de inclusão social, constata-se que existe um descompasso entre a conquista de direitos pela população e as reais e insuficientes condições para garanti-los. Uma das insuficiências é estrutural e organizacional e a outra é relacional. Comecemos pela estrutural e organizacional.

O SUS é o agente financiador da maioria dos atendimentos e das internações realizados no país, participando respectivamente em $61 \%$ e $70 \%$ destes, o que corresponde a uma significativa expansão de sua participação entre 1998 e 2003 (dados da Pesquisa Nacional por Amostra de Domicílios - Pnad), aumentando em 9,3\% e 4,1\%, respectivamente, para atendimentos e internações (Porto, Santos \& Ugá, 2006). Observou-se que o SUS financia preponderantemente populações de baixa renda já que sua participação no número de atendimentos e de internações em 2003 é predominante até o oitavo decil de renda da população. Ainda, como esperado, a participação do SUS é tanto maior quanto menor o nível de renda da população usuária (Porto, Santos \& Ugá, 2006).

Em relação ao tipo de oferta de serviço de saúde, a rede pública é composta principalmente por unidades de atenção básica (95\%) e serviços de emergência (65\%). Já a rede privada é composta por atenção especializada (74\%) e atenção hospitalar (79\%). Consequentemente existe uma maior utilização de serviços de baixa complexidade e de emergência pela população com menor renda, sugerindo menor acesso à atenção adequada (Almeida \& Travassos, 2000). Em contrapartida, há maior oferta de atendimento de alta complexidade ou alto custo pelo sistema público (Ribeiro et al., 2006). Existe, portanto, um esforço de ampliação do financiamento da saúde pelo SUS que já impacta alguns gargalos assistenciais históricos, apesar de persistir a falta de acesso às especialidade e aos serviços de investigação diagnóstica.

Os problemas de acesso refletem problemas de oferta, como discutido até o momento, e de organização dos serviços de saúde, que podem atuar como barreiras ao acesso. Esses aspectos põem em relevo a complexidade organizativa do sistema de saúde no Brasil, na qual o sistema público deve garantir a extensão de cobertura de serviços básicos e especializados para a população mais pobre, mas também a cobertura em procedimentos de alta 
complexidade para a maioria das camadas socioeconômicas (Ribeiro et al., 2006). Nesse contexto cresce a demanda por atendimento nos serviços hospitalares de emergência. Este comportamento pode apontar, ainda, que boa parte da população usuária do SUS busca assistência por meio de consultas de pronto atendimento nos prontos-socorros dos hospitais, em vez de buscar esse tipo de atendimento na rede básica de saúde que vem se ampliando a partir da implantação do Programa de Saúde da Família (PSF). Esses dados sugerem que os prontos-socorros ainda são importantes portas de entrada para assistência médica.

A pesquisa do Pnad reflete a complexidade do SUS, seus avanços, sua iniquidade, sua capacidade de oferta de serviços. Os desafios atuais do sistema de saúde brasileiro estão centrados na capacidade política e econômica dos atores sociais envolvidos na consolidação e implementação do SUS na administração de crises internas e externas, garantindo a sustentabilidade econômico-financeira do sistema, a cobertura da atenção à saúde da população com qualidade, eficiência e eficácia; e na percepção de que os resultados da reforma implementada no sistema de saúde brasileiro devem ser medidos em longo prazo (Soares, 2007). Portanto, o cenário de atendimento no SUS é de demanda reprimida, que aparece principalmente nos serviços de emergência hospitalar e de atenção básica, sem desconsiderar (e apesar de) os avanços já descritos.

Retomando a capacidade política dos atores envolvidos, que é necessária para a consolidação do SUS, a única opção política que viabiliza essa consolidação é a solidariedade com a população assistida, mesmo reconhecendo todas as dificuldades que o trabalhador do SUS enfrenta e a crônica falta de solidariedade com os mesmos. Os trabalhadores de saúde são produtos do sistema de relações em que estão mergulhados, mas são ao mesmo tempo, produtores deste mesmo sistema (Campos, G. W. S., 2006). O cenário instituído nos serviços de saúde em geral é de alienação, descompromisso, silêncio frente à impunidade, burocratização e desumanização. Em síntese, a ausência de instrumentos gerenciais eficazes de regulação e ordenamento da oferta que busquem viabilizar o acesso e a melhor utilização dos serviços do SUS por parte dos usuários, nos níveis macro e microorganizacionais, a desmotivação e o descompromisso dos trabalhadores de saúde com os resultados organizacionais, frutos, em parte, de uma gestão centrada nos meios e não nos resultados, contribuem de forma decisiva para a persistência de problemas relacionados à baixa efetividade do sistema de saúde brasileiro (Costa-e-Silva, Rivera \& Hortale, 2007).

Os baixos salários, as más condições de trabalho, a falta de instrumental adequado, a ausência de um plano de carreira, o excesso de demanda, a inadequada formação profissional, a concorrência não construtiva, a exigência de eficiência não sustentada, o autoritarismo, a verticalidade radical, a liderança indiferente ou ausente, a falta de comunicação ou suas distorções são as causas principais de deflagração de conflitos (Baremblitt, 2006).

Para romper com esse descrédito, as práticas nos serviços têm que melhorar, e a humanização deve estar contida em qualquer investimento em melhoria de qualidade dos serviços. A humanização pressupõe capacitação de profissionais, controle social e uma preocupação operativa com a reorganização dos serviços e das práticas. 
O aprimoramento da interação profissional/paciente é uma das facetas (somente uma delas!) da humanização da assistência (Nogueira-Martins, 2006). Para tal humanização são também importantes: contratação de profissionais suficientes para atender à demanda da população; aquisição de novos equipamentos médico-hospitalares; abertura de novos serviços de recuperação e adequação da estrutura física das instituições; revisão da formação dos profissionais com reestruturação dos currículos das faculdades da área da saúde; capacitação permanente dos profissionais; educação continuada; melhoria das condições de trabalho; e, por fim, melhoria da imagem do serviço público de saúde.

A integralidade como resposta governamental aos problemas de saúde esbarra na rede de serviços que não é suficiente no que diz respeito às especialidades. Esbarra na organização dos serviços já que, por melhor que seja o atendimento oferecido em um serviço, ele não pode dar conta de uma completa integralidade porque a integralidade depende da rede (integralidade ampliada) (Cecilio, 2001). Portanto a integralidade deve ser garantida num plano sistêmico, o das ações na rede de serviços (Pinheiro, 2001).

O conceito de integralidade remete, portanto, obrigatoriamente, ao de integração de serviços por meio de redes assistenciais, reconhecendo a interdependência dos atores e das organizações, em face da constatação de que nenhuma delas dispõe de recursos e competências suficientes para a solução dos problemas de saúde de uma população (Hartz \& Contandriopoulos, 2004). A integração consiste em coordenar as organizações autônomas para a realização de um projeto coletivo comum. Os discursos e as políticas sobre a integração fundamentam-se, assim, em três linguagens: a estrutural - modificação das fronteiras das organizações -, a clínica - modificações nas práticas profissionais - e a da cooperação - novos formatos de negociação e de acordos entre atores e organizações (Hartz \& Contandriopoulos, 2004). A instituição da rede exige estrutura organizacional, mas também exige modificação nos processos de trabalho e envolvimento cognitivo e afetivo dos profissionais.

É preciso enfrentar que a integralidade da atenção está fragilizada pelo padrão de assistência que é oferecido dentro da atual rede de serviços do SUS. Existe uma desvalorização velada das necessidades da população atendida e uma perversidade social que acaba por não solucionar problemas como carência de recursos humanos, ausência de diálogo com os trabalhadores, péssimas condições de trabalho e relação profissional/usuário no limite do aceitável.

Muito tem que ser feito para viabilizar um sistema de saúde integrado, porém esse investimento não pode depender só de recursos financeiros e projetos governamentais. Precisa-se de um novo processo de trabalho e do empenho particular de cada serviço (e por que não dizer de cada profissional) em reconhecer suas possibilidades de intervenção.

Existe uma aceitação implícita da baixa qualidade do serviço público por estar dirigido à população carente, que reconhece o serviço recebido como um favor e não como direito. A pertinência da questão da humanização da atenção está relacionada à concepção e aos valores que cada profissional tem a respeito de seu papel de profissional comprometido com as mudanças necessárias nesse campo (Traverso-Yépez \& Morais, 2004). 
Os direitos sociais não devem ser entendidos como uma ficção jurídica, apesar de existir uma dificuldade de transpor o direito jurídico para o direito realizado, devido à cultura política vivida pelo Brasil, patrimonialista e autoritária (Machado, Pinheiro $\&$ Guizardi, 2005). Como as mudanças de valores em uma sociedade ocorrem a partir de muita reflexão e exigem um longo tempo de maturação, é necessário investir na capacidade política dos atores da prática, em prol de uma postura mais solidária para enfrentar um cenário de direitos não realizados.

A valorização e a priorização da responsabilidade da pessoa, do zelo e da dedicação profissional por alguém são formas de superar as dicotomias entre oferta e demanda e entre postura técnica e relacional.

\section{As Intervenções Propostas pelo Ministério da Saúde no Intuito de Enfrentar as Dificuldades dos Serviços de Urgência e EMergênCia}

Para além do diagnóstico dos maus resultados do SUS, exaustivamente apontado pela academia, pelas corporações profissionais e pela imprensa, estratégias de enfrentamento têm sido propostas pelo Estado através de políticas de saúde.

As políticas de atenção às urgências nasceram em um cenário em que a prestação de serviços em melhores patamares de qualidade era uma preocupação explicitada na política de saúde do governo federal e em que havia muita insatisfação da população com os tradicionais serviços hospitalares. Em 2002, por solicitação do Conselho Nacional dos Secretários de Saúde (Conass) foi realizada a pesquisa Saúde na Opinião dos Brasileiros, na qual foram diagnosticadas as principais causas de descontentamento da população com os serviços de saúde. Muitas das queixas (baixa capacidade instalada, pouco acolhimento) diziam respeito aos serviços de emergência.

O resultado da pesquisa serviu de parâmetro para a elaboração do QualiSUS (Brasil/MS, 2004b), um conjunto de mudanças que visava a proporcionar maior conforto para o usuário, atendimento de acordo com o grau de risco, atenção mais efetiva pelos profissionais de saúde e menor tempo de permanência no hospital. Além de uma reforma mais estrutural dos hospitais, é criado um grupo de humanização do atendimento e há uma reorganização da oferta de leitos com priorização para a emergência, relevante porta de entrada para o sistema hospitalar. Outra importante política que visava à melhoria da qualidade da assistência é a Política Nacional de Humanização (Brasil/MS, 2003d) que se norteia pela valorização da dimensão subjetiva e social em todas as práticas de atenção e gestão no SUS. Para os serviços de urgência, propõe acolher a demanda e classificar as necessidades a partir da avaliação de riscos, garantindo o acesso e a referência aos demais níveis de atenção. Uma crítica feita a essa política é que a mesma se compromete como normatização, mas não garante o diálogo entre as normas e a realidade, crítica semelhante é realizada às éticas compassiva e utilitarista (Betts, 2006), nas quais os princípios são prioritários em relação aos sujeitos. 
Desde 2002 a área de urgência/emergência foi vista como um setor que exigia investimentos prioritários a partir de dois elementos centrais que geravam sobrecarga dos serviços disponibilizados para a população: a constatação do crescimento da demanda por serviços (aumento do número de acidentes e da violência urbana, e mudança do perfil demográfico e epidemiológico da população) e a estruturação insuficiente da rede assistencial (Moura, 2004).

Coube à gestão do ministro Borjas Negri a elaboração da portaria n. 2.048 (Brasil/ MS, 2002) que considerou as atribuições, o dimensionamento e a organização estrutural das diferentes unidades, bem como os aspectos da interação destas com os demais serviços. Seu principal mérito foi a definição das responsabilidades dos diferentes níveis de assistência na prestação do atendimento integral às urgências. Essa portaria já tratava de questões primordiais como a regulação, a capacitação profissional e a definição de recursos para os diferentes níveis de atenção.

Em 2003, o Ministério da Saúde cria, em seu organograma, a Coordenação Geral de Urgências/Emergência (CGUE) e assim foi constituído um grupo de profissionais que junto a representantes de outras entidades formataram a Política Nacional de Atenção às Urgências (Pnau) publicada por meio da portaria 1.863 (Brasil/MS, 2003a), com cinco pilares: promoção, organização da rede assistencial, capacitação de recursos humanos, regulação médica de urgência e humanização da assistência às urgências. A implantação da Pnau iniciou-se com o componente pré-hospitalar móvel (Brasil/MS, 2003b), por meio da implantação da Rede Nacional de Serviço de Atendimento Móvel de Urgência (Samu) - 192, com centrais de regulação médica de urgência (Brasil/MS, 2004a) em todo o país, visando à qualificação do acesso e à oferta de subsídios aos gestores para a tomada de decisões.

Na gestão do ministro Humberto Costa foram formuladas portarias que aprofundaram as atribuições específicas para a política como a regulação, a gestão, o acompanhamento e avaliação, além da capacitação. Para tal foi instituído o Comitê Gestor Nacional de Atenção às Urgências (Brasil/MS, 2003c), com perspectiva de planejamento de ações intersetoriais necessárias para a formatação do plano nacional de atenção às urgências. Houve ainda a criação de núcleos de educação em urgência com a proposição de grades curriculares para capacitação de recursos humanos da área e do Manual de Regulação Médica das Urgências (Brasil/MS, 2006c), elaborado como material de base oficial do ministério para os cursos de regulação médica de urgência de todo o território nacional.

Do conteúdo desse manual, vale destacar a pertinência do diagnóstico do cenário assistencial contido no capítulo. E como está a atenção às urgências nesse cenário? Esse diagnóstico retrata o baixo investimento em estratégias de promoção da qualidade de vida e saúde; o modelo assistencial ainda fortemente centrado na oferta de serviços e não nas necessidades dos cidadãos; a falta de acolhimento dos casos agudos de menor complexidade na atenção básica; a insuficiência de portas de entrada para os casos agudos de média complexidade; a má utilização das portas de entrada da alta complexidade; a insuficiência de leitos hospitalares qualificados, especialmente de Unidade de Tratamento Intensivo (UTI) 
e retaguarda para as urgências; as deficiências estruturais da rede assistencial - áreas físicas, equipamentos e pessoal; a inadequação na estrutura curricular dos aparelhos formadores; o baixo investimento na qualificação e educação permanente dos profissionais de saúde; as dificuldades na formação das figuras regionais e a fragilidade política nas pactuações; a incipiência nos mecanismos de referência e contrarreferência; as escassas ações de controle e avaliação das contratualizações externas e internas; e a falta de regulação.

Após a publicação (entre 2002 e 2005) dos documentos principais da Política Nacional de Atenção às Urgências que trataram da regulação e gestão, somaram-se duas portarias objetivando a expansão e estruturação desse nível de assistência. Em 2006 é publicada a portaria n. 3.125 (Brasil/MS, 2006b) que institui o Programa QualiSUS Urgência para qualificar a assistência nos componentes pré-hospitalar fixo e hospitalar; e em 2008 a portaria n. 2.922 (Brasil/MS, 2008a) que propõe a implantação/adequação de unidades de pronto atendimento (UPA) e salas de estabilização (SE) em locais/unidades estratégicos para a configuração de redes loco-regionais de atenção integral às urgências.

A gestão do ministro José Gomes Temporão não conseguiu avançar no desenvolvimento das proposições para a área, ainda muito incipientes, como a coordenação e capacitação/fixação dos profissionais. As UPAs e motolâncias (Brasil/MS, 2008b), instituídas na sua gestão, são estratégias de curto prazo (UPA) e complementares (motolâncias), que só foram possíveis a partir de um forte aporte de recursos federais. Como um condicionante positivo, as UPAs só podem ser projetadas para as áreas cobertas pelo Samu ou se houver concomitância de propostas de projetos Samu e UPA. Também é exigido um termo de compromisso de expansão da cobertura da estratégia de saúde da família atingindo no mínimo 50\% na área de abrangência de cada UPA, no prazo máximo de dois anos. Para o Rio de Janeiro, esse nível de expansão pretendida para o PSF não parece viável em um período de dois anos, em função da baixa cobertura no estado (24,7\%), em especial na capital, que chega a 3\%. Mas o condicionamento dessa expansão é um avanço inquestionável.

As UPAs foram implantadas como estratégia compensatória da insuficiência da atenção básica e da superlotação das emergências hospitalares no município do Rio de Janeiro, em especial entre os anos de 2007 e 2008. A primeira foi inaugurada em maio de 2007 e até abril de 2009 já haviam vinte UPAs inauguradas aqui. A previsão do governo do estado do Rio de Janeiro é abrir trinta UPAs até 2010, com capacidade para 12 mil atendimentos por mês em cada uma (Cortes, 2009).

Gostaria de destacar que esteve disponível no site do ministério, nas informações úteis no link do Samu (até o final de 2009) o áudio do discurso do ministro Temporão de lançamento das motolâncias, realizado em dezembro de 2008. Nele o ministro reafirma a importância que as políticas de urgência têm para o ministério, declarando que o Samu traz segurança e conforto para a população; o ministro também se compromete com a universalização do Samu até 2010. Cita o sucesso do Rio de Janeiro pela implantação das UPAs. Segundo o ministro, no começo do Governo Lula, o Samu era só uma ideia e hoje é uma realidade. Essa realidade tem sido pautada pelo ideal de serviços integrados em rede. 
A partir do site do Ministério da Saúde constatou-se que em 2004 haviam 23 Samu implantados e em 2009 eram 142. Nota-se então que apesar da prioridade dada ao Samu, ocorre um deslocamento de importância para a UPA, especialmente no plano político.

Mesmo exigindo um forte financiamento do nível federal, o atendimento às urgências é uma prioridade inegável para o atual governo. Há um forte investimento em recursos monetários e tecnológicos, porém, a normatização não possibilitou o avanço no quesito qualificação de profissionais (O 'Dwyer, 2009). Sua implantação tem sido a partir de uma plasticidade na forma de contrato dos profissionais que não favorece a sustentabilidade da política. Claro que esse problema não é exclusivo do Samu, UPAs e emergência hospitalar. A própria expansão do PSF, pretendida para o Rio de Janeiro, certamente sofrerá restrições pela gestão do trabalho.

Teoricamente, as políticas de saúde orientam as práticas, mas fatores sobre os quais os propositores de políticas não (ou pouco) interferem fazem com que as políticas aconteçam ou não. A formulação de política tem que ser entendida essencialmente como um processo político, em vez de um processo analítico de solução de problema. É um processo de negociação, barganha e acomodação de muitos diferentes interesses que refletem a ideologia do governo em poder (Walt, 1994).

Talvez a forma tradicional de fazer políticas idealize práticas em condições difíceis de realização. A não participação dos profissionais e das comunidades na formulação das políticas contribui com a distância entre o formulado e o implantado. Há necessidade de corresponsabilizar os profissionais e as comunidades na implantação e no controle de políticas e estratégias de saúde.

Outro problema que chama a atenção é a profusão de políticas que certamente confundem enormemente o gestor que se depara a todo o momento com novos programas, novas ações, devendo se readaptar constantemente sem, na maioria das vezes, participar do processo de formulação. Os usuários, por sua vez, mantêm-se à parte, geralmente desconhecendo por completo a existência dessas ações que, a princípio, deveriam favorecê-los (Almeida, 2005).

Uma crítica importante feita às políticas de saúde no Brasil é que o Ministério da Saúde faz um esforço grande na regulamentação de estratégias de curto prazo em atenção às propostas políticas de cada gestão de governo, em detrimento de políticas de médio e longo prazos, capazes de articular gestores estaduais e municipais e a sociedade civil, por mais de uma gestão de governo, em um projeto político para a saúde no país (Baptista, 2007).

Os instrumentos de indução política mais submetem os níveis subnacionais de governo às regras do sistema do que compõem uma política acordada e sustentada nas diversas realidades institucionais do país e respaldas numa prática social concreta. Essa forma de atuar se reforça e se mantém por um posicionamento também de acomodação das demais esferas de governo, que ainda percebem na relação de tutela com o Ministério da Saúde a garantia de benefícios e recursos para dar sustentação às suas políticas locais sem uma responsabilização maior de seus orçamentos próprios ou o compromisso com uma agenda política mais extensiva (Baptista, 2007). Vale dizer que uma política de âmbi- 
to nacional, ao estabelecer relações intergovernamentais, demanda a formulação de uma adequada estrutura de incentivos capaz de induzir os diversos atores a um comportamento cooperativo. Essa estrutura de incentivos se faz necessária porque estados e municípios, que são os agentes implementadores do SUS nos níveis regional e local, tendem a manter relações marcadas por disputas predatórias por recursos e ausência de comportamentos cooperativos (Viana, Faria \& Pacífico, 2006).

Independentemente das considerações feitas a respeito da dificuldade de implementação de políticas, o conjunto de normas que tratam da assistência às urgências e emergências tem o mérito de fazer um real diagnóstico das dificuldades desse nível de assistência, propor o envolvimento de toda a rede no enfrentamento do problema, de ser uma política de médio prazo ou longo e de ter mecanismos definidos de formação de profissionais. Claro que o investimento em capacitação não é privilégio para essa categoria específica de profissionais. Observa-se, a partir de 2003, uma maior valorização das políticas de recursos humanos do Ministério da Saúde, que se manifestou na criação da Secretaria de Gestão da Educação e do Trabalho em Saúde, cuja atuação tem-se orientado para a regulamentação e 'desprecarização' das condições de trabalho na área e para o fortalecimento da formação de profissionais para o SUS. A partir de 2007, são mais evidentes as estratégias de articulação entre os ministérios da Saúde e da Educação (Machado, Baptista \& Lima, 2007).

Percebi uma evolução na formulação da política, mas é necessário saber se essa evolução traduziu-se na prática dos níveis estaduais e municipais. Esse Estado que é indutor, formulador e regulador depende dos outros entes federativos autônomos para que a qualidade da assistência evolua, que é a expectativa principal gerada pelas políticas. Por isso discuto o estado do Rio de Janeiro, por suas peculiaridades na construção da assistência à saúde e sua importância para o país. Destaco como uma dessas peculiaridades, a gestão conjunta com a corporação dos bombeiros, especialmente na atenção às urgências ( $\mathrm{O}^{\prime}$ 'Dwyer, 2009).

\section{O Contexto de Atenção às UrgênCias e EmergênCias e O CAso do Estado do Rio de Janeiro}

Estou partindo dos pressupostos de que a insuficiência da rede contribui com a má qualidade da assistência às urgências e emergências e de que existe suficiência de políticas para nortear esse nível de atenção. Agora resta uma revisão sobre os problemas mais específicos desse nível de atenção e uma análise da situação do estado baseada em pesquisa empírica.

Considerando-se os tipos de patologias atendidas nas emergências, pode-se dizer que esses serviços são utilizados indevidamente no Brasil, pois cerca de $65 \%$ dos pacientes poderiam ser atendidos em ambulatórios (Lovalho, 2004). Entretanto, o problema não é exclusividade nossa. $O$ grande acúmulo de doentes nos serviços de emergência ocorre tanto no Brasil como no exterior, apesar das diferenças econômicas entre os países e entre as fontes de financiamento da assistência. Pacientes com problemas cuja solução é pertinente à atenção básica frequentam os serviços de emergência em vários países 
(Derlet \& Nishio, 1990; George, Read \& Westlake, 1993; Dale \& Green, 1995; Gill \& Rilley, 1996; Gill \& Reese, 1996; Shah \& Shah, 1996; Hotvedt et al., 1999; Krakau \& Hassler, 1999; Abdallat, Al-Smadi \& Abbadi, 2000; Fajardo-Ortiz \& Ramirez-Fernández, 2000; Lee, Lau \& Hazelett, 2000; Coleman, Irons \& Nicholl, 2001; Lee et al., 2001; Tannebaum \& Arnaold, 2001; Kalemoglu, Keskin \& Demirbas, 2004; Ruger, Richter \& Lewis, 2006; Dong et al., 2007; Hollander \& Pines, 2007).

Recentemente, Carret, Fassa e Domingues (2009) revisaram os estudos sobre a utilização inadequada de serviços de emergência. Esses autores reforçam que os serviços de emergência devem ser utilizados em circunstâncias específicas e que a utilização inadequada é prejudicial para os pacientes graves e para os não graves porque não acessam atendimento de seguimento na emergência. Indicam que o acesso à rede primária reduz o uso inapropriado de serviços de emergência se o paciente tiver rápido acesso ao atendimento de urgência na atenção básica (Carret, Fassa \& Domingues, 2009).

A resposta que os serviços oferecem para a população depende principalmente do tipo de atendimento que é demandado. Por um lado, a disponibilidade, o tipo, a quantidade de serviços e recursos (financeiros, humanos, tecnológicos), a localização geográfica, a cultura médica local, a ideologia do prestador, entre outros, são aspectos da oferta que influenciam o padrão de consumo de serviços de saúde. Por outro, as escolhas individuais também são cruciais, embora nem todas as necessidades se convertam em demandas e nem todas as demandas sejam atendidas. Inversamente, por produção de oferta, existe o uso de serviços não relacionado com as necessidades (Travassos \& Viacava, 2000).

Os pacientes que precisam de mais recursos tecnológicos em função da gravidade ou complexidade do seu problema de saúde são alvo de maior preocupação dos profissionais, dos gestores e dos formuladores de políticas de saúde. Os pacientes que exigem menos recursos tecnológicos são absorvidos como secundários, atendidos rapidamente após uma triagem e não são linearmente os que necessitam de menos atenção. Muitas vezes esses pacientes são os de menor recurso social e os que são pouco absorvidos pelos serviços de saúde. Entretanto a sua insistência em bater na porta dos hospitais de emergência continua a ser considerada como um erro a ser corrigido.

Pode-se imaginar que sem a revisão de todo o sistema de saúde, a emergência continuará a ser usada inapropriadamente pelos pacientes como alternativa para o atendimento primário. Essa constatação deve ser vista com cuidado para o paciente não ser rotulado como 'inapropriado' ao serviço que ele busca e sofrer a frustração de ter seu atendimento negado como eventualmente tem sido sugerido pela literatura (Derlet \& Nishio, 1990; Green \& Dale, 1992). Restrições de acesso têm sido propostas (McGugan \& Morrison, 2000; O 'Dwyer, Matta \& Pepe, 2008), mas são inaceitáveis na realidade do Brasil, um país com tanta desigualdade social.

No estado do Rio de Janeiro, no início do milênio, houve agravamento da crise política de planejamento e gestão vivenciadas nas interfaces das esferas federal, estadual e municipal. A crise agudizou-se entre os anos de 2004 e 2005 e foi consumada com a perda da gestão plena do município do Rio de Janeiro (modalidade de gestão definida na Norma 
Operacional Básica do SUS NOB SUS 01/96 - Brasil, 1997 - e na Norma Operacional da Assistência à Saúde (Noas) SUS 01/02 - Brasil, 2002), substituída no Pacto de Gestão do SUS pela assinatura do Termo de Compromisso de Gestão Municipal (portaria n. 399, de 22 de fevereiro de 2006) (Campos, P. C. C., 2006). Este termo foi assinado pelo estado do Rio de Janeiro em 2008.

A despeito das consequências da crise, os problemas persistiram em 2006, num cenário de mobilização institucional visando à melhoria das condições de atendimento com a incorporação de políticas como o QualiSUS, o Samu e o Projeto de Expansão e Consolidação da Saúde da Família (Proesf), iniciativa do Ministério da Saúde, apoiada pelo Banco Mundial (Bird), voltada para a organização e o fortalecimento da atenção básica à saúde no país (portaria n. 588, de 07 de abril de 2005 do Ministério da Saúde) (Campos, P. C. C., 2006).

No ano de 2005, portanto no auge da crise e no início das atividades do QualiSUS, (que no estado atuou em seis hospitais, sendo cinco no município do Rio de Janeiro), foi realizada uma pesquisa, financiada pelo Centro de Vigilância Sanitária da Secretaria de Saúde do Estado do Rio de Janeiro (CVS/SES-RJ), em trinta emergências hospitalares que compuseram uma amostra desses serviços no estado. Essa pesquisa analisou as práticas nos serviços hospitalares de emergência através da análise de conduta estratégica (Giddens, 1984). Construiu-se um roteiro a partir de instrumentos conhecidos, que foi validado por um painel de especialistas. Foram entrevistados noventa chefes médicos e enfermeiros das emergências.

O estudo registrou a relação dos serviços de emergência com usuários, com outros serviços do hospital e com a rede assistencial. Abordou a relação entre trabalhadores, a dimensão do seu quadro, de sua formação e de seus vencimentos. Relacionou a infraestrutura, cobrindo aspectos essenciais da organização, em que a assistência foi um dos componentes desse objeto mais amplo (O ’ Dwyer, Matta \& Pepe, 2008).

Pelo que foi discutido até então, vou utilizar os resultados dessa pesquisa para refletir sobre as causas referidas para a utilização indiscriminada das emergências hospitalares; sobre a carência de recursos humanos e estruturais; e, principalmente, sobre como se dá o relacionamento desses serviços com o resto da rede.

Nessa pesquisa a superlotação foi referida em $80 \%$ das emergências visitadas, tendo como principal causa o atendimento a pacientes com problemas ambulatoriais, informação que é compatível com a discussão realizada até o momento. Uma possível consequência é que os serviços que tendem a ser restritivos à demanda dos pacientes utilizam o discurso de que a emergência deve restringir seu atendimento ao paciente mais grave e eventualmente adotam uma política restritiva como efetivamente foi referido em uma emergência. Outros estudos brasileiros sobre os serviços de emergência têm demonstrado a dificuldade de lidar com a demanda excessiva de pacientes não graves (Jacobs \& Matos, 2005).

Outra questão prioritária para a descrição da situação da assistência às emergências no estado é a questão estrutural. Sabe-se da potência do parque hospitalar do Rio de Janeiro e do seu sucateamento ao longo dos anos. Na pesquisa do estado, em relação à estrutura dos 
hospitais visitados, havia uma maior insuficiência de recursos humanos para a realização da assistência ( $80 \%$ dos serviços) do que de recursos estruturais e tecnológicos (40\%). Esse aspecto é muito importante porque solucionar os problemas de estrutura predial tem sido mais viável politicamente do que enfrentar a carência numérica, a desqualificação, a alta rotatividade e o absenteísmo dos profissionais de saúde. Estratégias de contratação temporária têm sido utilizadas com resultados duvidosos (O ' Dwyer, Matta \& Pepe, 2008). A existência de vários tipos de vínculos, alguns temporários, dificulta a fixação do profissional e aumenta a necessidade de investir na qualificação deles. Alguns vínculos temporários são justificados por contratos emergenciais, mas precarizam a relação de trabalho, não resolvem a carência quantitativa de recursos humanos, diminuem o padrão qualitativo da equipe e não contribuem com a integração do corpo clínico da emergência com o do hospital.

Foram identificadas três situações de utilização dos serviços: o primeiro encontrado em $36 \%$ dos hospitais era de superlotação constante na emergência e de tempos de permanência muito grandes, tanto na emergência quanto na internação, com destaque para a clínica médica; o segundo, um cenário de utilização dos serviços de emergência e do leito hospitalar mais racional, perto de 100\% (em 20\% dos serviços); e o terceiro, encontrado em $44 \%$ dos serviços, em que havia subutilização da emergência e do leito hospitalar (O ' Dwyer, Matta \& Pepe, 2008). Essa heterogeneidade de utilização dos serviços hospitalares é compatível com uma estruturação da rede não planejada para racionalizar a utilização e o acesso aos serviços.

As dificuldades referidas com a rede (apontadas em todos os hospitais) tiveram como principais motivos: insuficiência da rede básica; falta de vagas para internação; falta de diálogo com o atendimento pré-hospitalar (Samu e bombeiros); falta de vaga para realização de exames complexos; indisponibilidade de ambulatórios especializados; falta de vagas em casas de apoio; e 'problemas sociais'. Foi encontrada em uma emergência uma situação muito irregular de um paciente 'internado' havia quatro anos.

Constatou-se como dado muito positivo, que a emergência traumática recebe um tratamento que responde às necessidades dos pacientes mais graves, refletindo positivamente nos indicadores de morbidade e mortalidade ( $O^{\prime}$ Dwyer, Matta \& Pepe, 2008). A percepção que os gerentes têm sobre a missão da emergência hospitalar é que seu atendimento deve limitar-se às urgências e emergências definidas tecnicamente. Apenas um dos chefes de serviço entrevistado reconheceu a necessidade de acolher todas as demandas que aparecem nos serviços.

Corresponder às expectativas da clientela é um dos maiores desafios para a organização da assistência (Pinheiro, 2001). Esse desafio deve ser perseguido por gestores e acolhido pelos profissionais, já que são os atores da prática que legitimam o SUS perante a população.

Outra pesquisa, realizada em um grande hospital do município do Rio de Janeiro (Campos, P. C. C., 2006), reflete os desafios de um atendimento centrado nos hospitais, com baixa responsabilização pelo encaminhamento do paciente e sua consequente 'peregrinação'. Conclui que a baixa cobertura do PSF, o ‘hospitalocentrismo’ de emergência, o destrato no acesso, a pouca credibilidade nos atendimentos médicos e a invisibilidade do 
usuário/paciente configuram um sistema indutor de peregrinações no SUS. Desse modo, sobrepõe, paradoxalmente, violência ao cuidado (Campos, P. C. C., 2006).

Outra pesquisa também realizada em uma emergência do município do Rio de Janeiro (Deslandes, 2007) tratou da banalização do sofrimento do usuário. Concluiu que: o sofrimento do outro é lido como menor, suportável, menos grave; o outro é destituído de atributos considerados merecedores de atenção, o outro e tudo que lhe diz respeito é banalizado, inclusive seu sofrimento; o sofrimento do outro raramente mobiliza vínculos empáticos porque o sofrimento do cuidador também foi banalizado; o sofrimento do outro se torna meramente um contexto usual, naturalizado no modelo cultural de cuidados médicos profissionais (regido pela racionalidade biomédica tradicional). Ou seja, o profissional nega o outro, por atribuição de menor status social ou moral, desmerecendo seu sofrimento.

Esses trabalhos (Skaba, 1997; Campos, P. C. C., 2006; O’Dwyer, Matta \& Pepe, 2008) mostram a precária assistência para um grupo de pacientes que acessa a rede através dos hospitais, mas não resolve seu problema. Do total de pacientes que poderiam ser atendidos em ambulatórios e preferem eleger a emergência como porta de entrada, $36 \%$ não dão seguimento ambulatorial à patologia que motivou a procura ao serviço de emergência (Lovalho, 2004), o que corrobora a necessidade de acompanhamento desses pacientes, para garantir a efetividade da assistência. Fortalecer a atenção básica como porta de entrada do sistema, cumprir o atendimento de urgências previsto para ser realizado no âmbito da atenção básica são ações que encontram resistência e que a longo prazo podem mudar o perfil do paciente que demanda atendimento na emergência. $\mathrm{Na}$ prática não houve ainda essa mudança (Oliveira, 2004). O contexto da assistência nas emergências hospitalares do Rio foi anteriormente analisado por Skaba (1997) demonstrando muitas semelhanças.

$\mathrm{Na}$ maioria dos estados e municípios, os serviços de emergência sofrem o maior impacto da desorganização do sistema, tornando-se o alvo preferido para as críticas ao modelo nacional de assistência à saúde (Scarpelini, 2007). Em um país como o nosso, o caminho para superar o modelo atual de atenção nos serviços de emergência deverá ser de caráter sistêmico e ter como foco o usuário, com redefinição e integração das vocações assistenciais, reorganização de fluxos e repactuação do processo de trabalho (Bittencourt \& Hortale, 2007).

No âmbito internacional, muito países desenvolveram sistemas para atendimento às urgências/emergências. Contudo, embora atualmente exista uma grande experiência acumulada no atendimento às urgências/emergências, também existe uma diversidade de modelos. As principais diferenças nos modelos estão na presença de profissionais com especialidade em emergência nos hospitais e na centralidade do profissional médico ou paramédico para o atendimento pré-hospitalar. No modelo francês, o médico é responsável pelo atendimento pré-hospitalar que é mais resolutivo, já no modelo americano, paramédicos transportam o paciente para os hospitais o mais rápido possível. Na verdade, ainda existem dúvidas sobre qual o melhor modelo de organização dos sistemas de urgência no 
mundo, contudo não se contesta a necessidade dessa organização. Embora existam particularidades entre os modelos atuais, todos apresentam, em geral, bons resultados (Scarpelini, 2007). Este é mais um argumento para o SUS investir na reorganização e regulação desses serviços, como proposto na política de urgência.

Como já dito, a Pnau foi uma estratégia de qualificação da assistência às urgências, e o estado do Rio de Janeiro, já privilegiado pelo QualiSUS, abrigou o primeiro Samu regionalizado do país. Entre 2005 e 2006 foram implantados os três Samu do estado que assistem a $75 \%$ da população (O 'Dwyer, 2009). São Samu metropolitanos: o Samu Rio, para a capital; o Samu Metropolitano I, que atende a dez municípios; e o Samu Metropolitano II, que atende a sete municípios. Esse investimento para a área de urgência ocorreu em um cenário de precariedade da atenção básica. Em pesquisa recente realizada sobre o desempenho dos Samu no estado do Rio de Janeiro (O'Dwyer, 2009) foram evidenciados: a dificuldade de acesso à atenção básica (como para consultas de pré-natal e atendimento a diabéticos); a inoperância das referências hospitalares; e a dificuldade de continuidade de atendimento na atenção básica, uma constatação coerente com a discussão sobre superlotação nas emergências. A precariedade na gestão de recursos humanos esteve implicada com os problemas evidenciados na pesquisa, assim como insuficiências estruturais diversas ( $\mathrm{O}^{\prime}$ Dwyer, 2009). Como já abordado, a UPA foi a estratégia escolhida para compensar o déficit na atenção básica para o estado e atualmente existe um real investimento para ampliação da saúde da família.

O desafio é fazer funcionar o SUS com todo seu potencial de qualidade, em particular no Rio de Janeiro, dada sua capacidade instalada e a repercussão para o restante do país (Bittencourt \& Hortale, 2007).

\section{CONCLUSÃo}

A insuficiência do atendimento de especialidades e de investigação diagnóstica gera um grande estrangulamento na assistência que inviabiliza a integralidade e produz uma grande parte das pequenas urgências, compondo o grupo que superlota as emergências e a atenção básica. Outras necessidades, produzidas sob a ótica do paciente e não atendidas principalmente por falta de vínculos estabelecidos na atenção básica, também superlotam as emergências.

Os serviços de emergência programam seu atendimento no sentido de atender urgências e emergências médicas. Recebem uma demanda espontânea de atendimento não emergencial acreditando que estão atendendo a uma demanda que não é sua - do serviço de emergência - e sim da atenção básica.

Cria-se um ambiente desfavorável e eventualmente até hostil para o atendimento demandado pelos indivíduos nos dois principais espaços de assistência no SUS. Na emergência, por esse tipo de atendimento ser considerado 'inadequado', e na atenção básica, por ser menos valorizado do que as demandas programáticas e as ações de prevenção e promoção da saúde. 
É preciso reconhecer que o padrão de assistência oferecido dentro da atual rede de serviços do SUS está fragilizado. Existe uma desvalorização velada das necessidades da população atendida e uma perversidade social que acaba por não solucionar problemas como carência de recursos humanos, ausência de diálogo com os trabalhadores, péssimas condições de trabalho e relação profissional/usuário no limite do aceitável.

A não assistência do que é considerado essencial para a saúde da população, a desigualdade social entre quem recebe e quem presta e gerencia os serviços de saúde, a dificuldade, no dia a dia, de exercer o controle social, e mesmo da participação popular no monitoramento das instituições comprometidas com a saúde, são questões complexas e de difícil abordagem. É preciso construir alianças entre os usuários e profissionais de saúde para o enfrentamento dos determinantes sociais de doença, das desvantagens sociais enfrentadas pela população e sua dificuldade de mobilidade social. É a partir dessa aliança que os problemas da assistência prestada pelos serviços de emergência devem ser vistos, com o propósito de aumentar a regulação e direcionar os pacientes depois do acesso a qualquer porta de entrada do SUS. Trata-se do desafio da gestão assistencial com grande integração da rede.

Os interesses dos gestores, profissionais e população têm que ser conciliados na construção de um sistema de saúde estruturalmente compatível com sua missão e sensível às demandas e necessidades da população - as biológicas e as percebidas como sociais. Interesses comuns que rompam com a 'neutralidade secular de exclusão' de muitos e com o 'desentendimento profundo' que ocorre nos serviços do SUS. Essas expressões são fruto de uma pesquisa de campo em um serviço de emergência onde houve descompasso manifesto entre as expectativas e diferentes percepções de urgência dos especialistas e dos pacientes (Giglio-Jacquemot, 2005).

Há necessidade das políticas públicas articularem intervenções mais efetivas já que as vigentes definem a configuração dos territórios sanitários com suas redes hierarquizadas e solidárias (Brasil/MS, 2006a), mas as experiências concretas ainda não praticam a solidariedade. O Pacto objetiva a solidariedade e a cooperação entre os entes federativos para em vez de pactuar 'procedimentos', pactuar 'responsabilidades'.

A ordenação do acesso à rede assistencial de urgência por meio da regulação médica pode transformar o cenário dos hospitais de referência em urgência e induzir a reorganização, com aumento da resolutividade e da qualidade dos serviços (Santos, 2002).

A regulação assim como a organização da rede em níveis com a referência e contrarreferência sugerem (e/ou determinam) que a população compreenda e siga as regras de acesso ao sistema, mas também é necessário flexibilizá-las, criá-las e segui-las com uma lógica mais compativel com os anseios dos usuários.

De forma geral, mesmo em redes normatizadas, com fluxos formais de referência e contrarreferência, a circulação de usuários é obstaculizada por inúmeros procedimentos burocráticos, por competições entre especialidades profissionais e por uma gama de 'sentidos' que a referência e a contrarreferência têm para os profissionais que devem acolher esses usuários e que têm o seu processo de trabalho determinado pelo apelo da 
tecnologia e/ou do procedimento e não da necessidade do usuário. Na verdade, os serviços realizam um trabalho solitário e de isolamento dentro da rede já que desconsideram as dificuldades dos outros serviços que se traduzem na não aceitação do paciente sem considerar outros acessos negados. Não há maleabilidade no tratamento dispensado às normas e sim um apego à missão do serviço, que desconsidera outras barreiras e a noção de rede assistencial.

Um modo de organizar a rede de serviços de saúde sugerida é uma reorganização orientada pelo direito ao acesso e pela integralidade: primeiro, os modelos de organização da rede devem levar em conta os territórios da vida social; segundo, deve-se abandonar a ideia de porta de entrada única; terceiro, a proposta da rede regionalizada e hierarquizada só é boa à medida que facilite o atendimento integral das necessidades das pessoas; e quarto, os profissionais devem estar disponíveis para serem portas de entrada (Mattos, 2007).

A inflexível definição de porta de entrada do sistema de saúde pela atenção básica com referência territorial, em vez de promover inclusão, dificulta o acesso aos outros serviços de saúde (Oliveira, 2004). Essa perda de liberdade em relação à busca por assistência contraria princípios democráticos e destitui o usuário de sua identidade de cidadão e de seu papel ativo como agente intencional na defesa de seus interesses e na livre decisão do melhor modo de satisfazer suas necessidades. Não se trata de desconsiderar a relevância da base territorial como referência para o estabelecimento de vínculos profissional/população. $\mathrm{O}$ que se defende é que, em relação à demanda espontânea, o sistema deve estar plenamente aberto ao seu acolhimento, seja em que nível e em que serviço for (Oliveira, 2004).

O investimento em políticas de urgência e emergência reflete a necessidade de mudar o atual quadro que gera enorme desgaste dos profissionais e dos usuários. A recente pesquisa de (Carret, Fassa \& Domingues, 2009) aponta para a importância do atendimento de pequenas urgências pela atenção básica, o que foi previsto por portaria desde 2002, sendo reforçado pelas recentes portarias que condicionam os projetos das UPAs à expansão do Samu e PSF, integrando a atenção às urgências.

Mas além das normatizações necessárias para as mudanças, ações políticas e éticas são necessárias para a transformação das práticas nos serviços. Deve haver solidariedade entre os gestores municipais e estaduais, entre os gestores dos serviços de emergência e da atenção básica e entre os médicos do Samu e reguladores médicos, que são os responsáveis sanitários pela integração da rede assistencial. Solidariedade entre profissionais e entre eles e a população. Os usuários devem usufruir realmente dos seus direitos de cidadãos.

O que está em jogo é a definição do que é responsabilidade de cada serviço e de cada profissional diante das diferentes demandas. Na saúde, a responsabilidade é tensionada pela crescente demanda gerada pelo conhecimento biomédico (demanda construída em torno do complexo industrial da saúde exigindo sempre a oferta de mais serviços e mais diferenciada) e por questões mais amplas que interferem na saúde (como a violência) e que exigem uma posição política da sociedade para seu enfrentamento.

O enfrentamento político do que é demanda por atenção médica nessa complexa ordem social médica não será objeto de maior reflexão neste capítulo, mas foi citado para 
reforçar que as práticas nos serviços de saúde precisam ser qualificadas como um imperativo ético de um SUS legitimado por ser equitativo, universal e integral.

A legitimação e a sustentabilidade política do SUS surgem a partir da confiança do usuário nos seus serviços; confiança que precisa de experiências positivas para ser construída.

\section{ReFERÊNCIAS}

ABDALLAT, A. M.; AL-SMADI, I. \& ABBADI, M. D. Who uses the emergency room services? Eastern Mediterranean Health Journal, 6(5): 1.126-1.129, 2000.

ALMEIDA, C. \& TRAVASSOS, C. Health sector reform in Brazil: a case study of inequity. International Journal of Health Services, 30(1): 129-162, 2000.

ALMEIDA, E. C. Humanização e Politicas de Saúde: um estudo sobre o usos e sentidos das propostas de humanização nas políticas de atenção à saúde da mulher, 2005. Tese de Doutorado, Rio de Janeiro: Instituto de Medicina Social, Universidade Estadual do Rio de Janeiro.

BAPTISTA, T. W. F. Análise das portarias ministeriais da saúde e reflexões sobre a condução nacional da política de saúde. Cadernos de Saúde Pública, 23(3): 615-626, 2007.

BAREMBLITT, G. Conflitos e malestares na prática hospitalar. Portal Humaniza. Disponível em: <www.portalhumaniza.org.br/ph/texto.asp?id=36>. Acesso em: 8 nov. 2006.

BETTS, J. Considerações sobre o que é humano e o que humanizar. Portal Humaniza. Disponível em: 〈www.portalhumaniza.org.br/ph/texto.asp?id=37>. Acesso em: 8 nov. 2006.

BITTENCOURT, R. J. \& HORTALE, V. A. A qualidade nos serviços de emergência de hospitais públicos e algumas considerações sobre a conjuntura recente no município do Rio de Janeiro. Revista Ciência Ë Saúde Coletiva, 12(4): 929-934, 2007.

BRASIL. Ministério da Saúde. Portaria n. 2.048, de 05 nov. 2002. Aprova o Regulamento Técnico dos Sistemas Estaduais de Urgências e Emergências. Diário Oficial da União, Brasília, 12 nov. 2002.

BRASIL. Ministério da Saúde. Portaria n. 1.863, de 29 set. 2003. Institui a Política Nacional de Atenção às Urgências. Diário Oficial da União, Brasília, 06 out. 2003 a.

BRASIL. Ministério da Saúde. Portaria n. 1.864, de 29 set. 2003. Institui o Componente préhospitalar móvel da Pnau - o Samu. Diário Oficial da União, Brasília, 06 out. 2003b.

BRASIL. Ministério da Saúde. Portaria n. 2.072, de 30 out. 2003. Institui o Comitê Gestor Nacional de Atenção às Urgências. Diário Oficial da União, Brasília, 31 out. 2003c.

BRASIL. Ministério da Saúde. Política Nacional de Humanização da Atenção e da Gestão em Saúde no Sistema Único de Saúde - SUS. Brasília: Ministério da Saúde, 2003d.

BRASIL. Ministério da Saúde. Portaria n. 2.657, de 16 dez. 2004. Estabelece as atribuições das Centrais de Regulação Médica de Urgências. Diário Oficial da União, Brasília, 17 dez. 2004a.

BRASIL. Ministério da Saúde. Projeto de Investimento na Qualificação do SUS - QualiSUS. Brasília, 2004b.

BRASIL. Ministério da Saúde. Portaria n. 399, de 22 fev. 2006. Diretrizes do Pacto pela Saúde Consolidação do Sistema Único de Saúde. Diário Oficial da União, Brasília, 23 fev. 2006a.

BRASIL. Ministério da Saúde. Portaria n. 3.125, de 7 dez. 2006. Institui o QualiSUS -Urgência. Diário Oficial da União, Brasília, 08 dez. 2006b.

BRASIL. Ministério da Saúde. Regulação Médica das Urgências: normas e manuais técnicos do Ministério da Saúde. Brasília: Ministério da Saúde, 2006c.

BRASIL. Ministério da Saúde. Portaria n. 2922, de 2 dez. 2008. Propõe a implantação/adequação de Unidades de Pronto Atendimento - UPA. Diário Oficial da União, Brasília, 03 dez. 2008a. 
BRASIL. Ministério da Saúde. Portaria n. 2.971, 8 dez. 2008. Institui o veículo motocicleta motolância. Diário Oficial da União, Brasília, 09 dez. 2008b.

CAMPOS, G. W. S. Subjetividade e administração de pessoal: considerações sobre modos de gerenciar o trabalho em equipes de saúde. In: MERHY, E. E. (Org.). Agir em Saúde: um desafio para o público. 2. ed. São Paulo: Hucitec, 2006.

CAMPOS, P. C. C. 'Peregrinação' no Sistema Único de Saúde: experiência da sala de acolhimento na porta de entrada hospitalar, 2006. Dissertação de Mestrado, Rio de Janeiro: Instituto Oswaldo Cruz, Fundação Oswaldo Cruz.

CARRET, M. L. V.; FASSA, A. G. \& DOMINGUES, M. R. Prevalência e fatores associados ao uso inadequado do serviço de emergência: uma revisão sistemática da literatura. Cadernos de Saúde Pública, 25(1): 7-28, 2009.

CECILIO, L. C. O. As necessidades de saúde como conceito estruturante na luta pela integralidade e equidade na atenção em saúde. In: PINHEIRO, R. \& MATTOS, R. A. (Orgs.). Os Sentidos da Integralidade na Atenção e no Cuidado à Saúde. Rio de Janeiro: IMS/Uerj, Abrasco, 2001.

COLEMAN, P.; IRONS, R. \& NICHOLL, J. Will alternative immediate care services reduce demands for non-urgent treatment at accident and emergency? Emergency Medicine Journal, 18: 482-487, 2001.

CORTES, S. Superlativo de acertos. O Globo, Rio de Janeiro, 23 mar. 2009. Opinião, p. 7.

COSTA-E-SILVA, V.; RIVERA, F. J. U. \& HORTALE, V. A. Projeto Integrar: avaliação da implantação de serviços integrados de saúde no Município de Vitória, Espírito Santo, Brasil. Cadernos de Saúde Pública, 23(6): 1.405-1.414, 2007.

DALE, J. \& GREEN, J. Primary care in the accident and emergency department: I. Prospective identification of patients. BMJ, 12(311): 423-426, 1995.

DERLET, R. W. \& NISHIO, D. A. Refusing care to patients who present to an emergency department. Annals of Emergency Medicine, 19(3), supl.: 262-267, 1990.

DESLANDES, S. F. O cuidado humanizado como valor e ethos da prática em saúde. In: PINHEIRO, R. \& MATTOS, R. A. (Orgs.). Razões Públicas para a Integralidade em Saúde: o cuidado como valor. Rio de Janeiro: Cepesc, IMS/Uerj, Abrasco, 2007.

DONG, S. L. et al. Predictive validity of a computerized emergency triage tool. Academic Emergency Medicine, 14(1): 16-21, 2007.

FAJARDO-ORTIZ, G. \& RAMIREZ-FERNÁNDEZ, F. A. Utilización del servicio de urgências em um hospital de especialidades. Cirurgía y Cirujanos, 68: 164-168, 2000.

GEORGE, S.; READ, S. \& WESTLAKE, L. Differences in priorities assigned to patients by triage nurses and by consultant physicians in accident and emergency departments. Journal of Epidemiology and Community Health, 47(4): 312-315, 1993.

GIDDENS, A. A Constituição da Sociedade. São Paulo: Martins Fontes, 1984.

GIGLIO-JACQUEMOT, A. Urgências e Emergências em Saúde: perspectivas de profissionais e usuários. Rio de Janeiro: Editora Fiocruz, 2005.

GILL, J. M. \& REESE, C. L. Disagreement among health care professionals about urgent care needs of emergency. Annals of Emergency Medicine, 28(5): 474-479, 1996.

GILL, J. M. \& RILLEY, A. W. Nonurgent use of hospital emergency departments; urgency from patient's perspective. The Journal of Family Practice, 42(5): 491-496, 1996.

GREEN, J. \& DALE, J. Primary care in accident and emergency and general practice; a comparison. Social Science EO Medicine, 35(8): 987-995, 1992.

HARTZ, Z. M. A \& CONTANDRIOPOULOS, A. P. Integralidade da atenção e integração de serviços de saúde: desafios para avaliar a implantação de um "sistema sem muros". Cadernos de Saúde Pública, 20, supl. 2: 5.331-5.336, 2004. 
HOLLANDER, J. E. \& PINES, J. M. The emergency department crowding paradox: the longer you stay, the less care you get. Annals of Emergency Medicine, 50(5): 497-499, 2007.

HOTVEDT, R. et. al. How urgent are emergency admissions? An evaluation of emergency admission to general hospitals in a Norwegian county. International Journal of Technology Assesment in Health Care, 15(4): 699-708, 1999.

JACOBS, P. C. \& MATOS, E. P. Estudo exploratório dos atendimentos em unidade de emergência em Salvador - Bahia. Revista da Associação Médica Brasileira, 51(6): 348-353, 2005.

KALEMOGLU, M.; KESKIN, O. \& DEMIRBAS, S. et al. Non-urgent patients in an emergency medical service. Revista Médica de Chile, 132: 1.085-1.087, 2004.

KRAKAU, I. \& HASSLER, E. Provision for clinic patients in the ED produces more nonemergency visits. American Journal of Emergency Medicine, 17(1): 18-20, 1999.

LEE, A.; LAU, F. L. \& HAZELETT, C. B. Factors associated with non-urgent utilization of accident and emergency services: a case-control study in Hong Kong. Social Science $\mathcal{E}$ Medicine, 51(7): 1.075$1.085,2000$.

LEE, A. et al. Morbidity patterns of non-urgent patients attending accident and emergency departments in Hong Kong: cross-sectional study. Hong Kong Medical Journal, 7(2): 131-138, 2001.

LOVALHO, A. F. Administração de serviços de saúde em urgências e emergências. O Mundo da Saúde, 28(2): 160-171, 2004.

LUZ, M. T. Ordem Social, Instituições e Políticas de Saúde no Brasil: textos reunidos. Rio de Janeiro: Cepesc, IMS/Uerj, Abrasco, 2007.

MACHADO, C. V.; BAPTISTA, T. W. F. \& LIMA, L. D. Responsabilidade do Estado, papel e relações entre Poderes na saúde: duas ou três coisas que sabemos disso. In: PINHEIRO, R. \& MATTOS, R. A. (Orgs.). Razões Públicas para a Integralidade em Saúde: o cuidado como valor. Rio de Janeiro: Cepesc, IMS/Uerj, Abrasco, 2007.

MACHADO, F. R. S.; PINHEIRO, R. \& GUIZARDI, F. L. Direito à saúde e integralidade no SUS: o exercício da cidadania e o papel do Ministério Público. In: PINHEIRO, R. \& MATTOS, R. A. (Orgs.). Construção Social da Demanda: direito à saúde, trabalho em equipe e participação e espaços públicos. Rio de Janeiro: IMS/Uerj, Abrasco, 2005.

MATTOS, R. A. Repensando a organização da rede de serviços de saúde a partir do princípio da integralidade. In: PINHEIRO, R. \& MATTOS, R. A. (Orgs.). Razões Públicas para a Integralidade em Saúde: o cuidado como valor. Rio de Janeiro: Cepesc, IMS/Uerj, Abrasco, 2007.

McGUGAN, E. A. \& MORRISON, W. Primary care or A \& E? A study of patients redirect from accident \& emergency departments. Scottish Medical Journal, 45(5): 144-147, 2000.

MOURA, I. R. Avaliação da Organização Assistencial das Unidades Hospitalares de Pronto Atendimento no Município de Goiânia, Adotando como Referência a Política Nacional de Atenção às Urgências, 2004. Dissertação de Mestrado, Rio de Janeiro: Escola Nacional de Saúde Pública Sergio Arouca, Fundação Oswaldo Cruz.

NOGUEIRA-MARTINS, M. C. F. Oficinas de humanização: fundamentação teórica e descrição de uma experiência com um grupo de profissionais da saúde. In: DESLANDES, S. F. (Org.). Humanização dos Cuidados em Saúde: conceitos, dilemas e práticas. Rio de Janeiro: Editora Fiocruz, 2006.

O ' DWYER, G. O Princípio da Integralidade e o SAMU, 2009. Tese de Doutorado, Rio de Janeiro: Instituto de Medicina Social, Universidade de Estado do Rio de Janeiro.

O ’ DWYER, G.; MATTA, I. E. A. \& PEPE, V. L. E. Avaliação dos serviços hospitalares de emergência do estado do Rio de Janeiro. Revista Ciência E⿱ Saúde Coletiva, 13(5): 1.637-1.648, 2008.

OLIVEIRA, L. H. Cidadãos Peregrinos: os "usuários" do SUS e os significados de sua demanda a prontosocorros e hospitais no contexto de um processo de reorientação do modelo assistencial. Uma análise a partir 
de usuários do SUS no município de Juiz de Fora, 2004. Tese de Doutorado, Rio de Janeiro: Instituto de Medicina Social, Universidade de Estado do Rio de Janeiro.

PINHEIRO, R. As práticas no cotidiano na relação oferta e demanda dos serviços de saúde: um campo de estudo e construção da integralidade. In: PINHEIRO, R. \& MATTOS, R. A. (Orgs.). Os Sentidos da Integralidade na Atenção e no Cuidado à Saúde. Rio de Janeiro: IMS/Uerj, Abrasco, 2001.

PORTO, S. M.; SANTOS, I. S. \& UGÁ, A. D. A utilização de serviços de saúde por sistemas de financiamento. Revista Ciência Ë Saúde Coletiva, 11(4): 895-910, 2006.

RIBEIRO, M. C. S. A. et al. Perfil sócio-demográfico e padrão de utilização de serviços de saúde para usuários e não-usuários do SUS - Pnad 2003. Revista Ciência $\mathcal{E}$ Saúde Coletiva, 11(4): 1.011$1.022,2006$.

RUGER, J. P.; RICHTER, C. J. \& LEWIS, L. M. Clinical and economic factors associated with ambulance use to the emergency department. Academic Emergency Medicine, 13(8): 879-885, 2006.

SANTOS, J. S. Da fundação do Hospital das Clínicas à criação da unidade de emergência e sua transformação em modelo nacional de atenção hospitalar às urgências. Medicina, 35: 403- 418, 2002.

SCARPELINI, S. A organização do atendimento às urgências e trauma. Medicina, 40(3): 315-320, 2007.

SHAH, N. M. \& SHAH, M. A. Predictors of non-urgent utilization of hospital emergency services in Kuwait. Social Science EO Medicine, 42(9): 1.313-1.323, 1996.

SOARES, A. Formação e desafios do sistema de saúde no Brasil: uma análise de investimentos realizados para ampliação da oferta de serviços. Cadernos de Saúde Pública, 23(7): 1.565-1.572, 2007.

TANNEBAUM, R. D. \& ARNAOLD, J. L. Emergency medicine in southern Brazil. Annals of Emergency Medicine, 37(2), supl.: 223-228, 2001.

TRAVASSOS, C. \& VIACAVA, F. Desigualdades geográficas e sociais na utilização de serviços de saúde no Brasil. Revista Ciência E Saúde Coletiva, 5(1): 133-149, 2000.

TRAVERSO-YÉPEZ, M. \& MORAIS, N. A. Reivindicando a subjetividade dos usuários da Rede Básica de Saúde: para uma humanização do atendimento. Cadernos de Saúde Pública, 20(1): 80-88, 2004.

VIANA, A. L. D.; FARIA, R. \& PACÍFICO, H. O SUS e as novas práticas de avaliação para gestão em redes: é possível conciliar a visão clássica de avaliação como novas práticas? In: PINHEIRO, R. \& MATTOS, R. A. (Orgs.). Gestão em Redes: práticas de avaliação, formação e participação na saúde. Rio de Janeiro: IMS/Uerj, Abrasco, 2006.

WALT, G. Health Policy: an introduction to process and power. Johannesburg: Witwatersrand University Press, 1994. 